\title{
Percepções dos profissionais de educação física das academias de Montes Claros-MG acerca da conduta na pós-reabilitação da lesão Condromalácia Patelar
}

\author{
Perceptions of physical education professionals at the Montes Claros-MG academies \\ about the post-rehabilitation conduct of the chondromalacia patellar injury
}

Percepciones de los profesionales de educación física en las academias Montes ClarosMG sobre la conducta posterior a la rehabilitación de la lesión patelar de condromalacia

Camila Fernanda Santos Oliveira ${ }^{1 \star}$, André Demian dos Santos ${ }^{2}$, Saulo Daniel Mendes Cunha ${ }^{3}$.

\section{RESUMO}

Objetivo: Analisar a percepções dos profissionais de educação física sobre a lesão Condromalácia Patelar, bem como a conduta clínica para o tratamento desta patologia. Métodos: A pesquisa foi descritiva e de corte transversal, desenvolvida por abordagem qualitativa. Foram entrevistados 12 profissionais formados no curso de educação física - Bacharelado, atuantes como instrutor/personal na cidade Montes Claros-MG. A coleta de dados foi realizada entre os meses de agosto e setembro de 2019. Teve como instrumento a entrevista individual com roteiro semiestruturado, onde foi transcrita na íntegra e, após transcrição e leitura dos relatos, utilizando o método da categorização como coleta dos dados. Resultados: As ideias consideradas mais relevantes da entrevista foram selecionadas e agrupadas em quatro categorias. Foi possível evidenciar que os profissionais, parecem não apresentar um conhecimento satisfatório da Condromalácia Patelar, visto que, apresentaram respostas superficiais ou que não condiz com a literatura presente. Conclusão: Conclui-se que apesar da pós-reabilitação ser uma área de importante atuação para o campo da educação física, ainda falta o conhecimento necessário para atuação dos mesmos, podendo agravar, ou não, o quadro patológico do cliente. Nessa perspectiva, futuros estudos devem ser realizados para investigação da Condromalácia Patelar e outras lesões relacionadas a saúde/reabilitação.

Palavras-chave: Profissionais de educação física, Condromalácia patelar, Pós-reabilitação.

\begin{abstract}
Objective: To analyze the perceptions of Physical Education professionals about Chondromalacia Patellar lesion, as well as the clinical conduct for the treatment of this pathology. Methods: The research was descriptive and cross-sectional, developed by qualitative approach. Twelve professionals who graduated from the Physical Education - Bachelor Degree course, who worked as instructor / personal in the city Montes Claros-MG, were interviewed. Data collection was performed between August and September 2019. The instrument was the individual interview with semi-structured script, which was transcribed in full and, after transcription and reading of the reports, using the categorization method as data collection. Results: The most relevant interview ideas were selected and grouped into four categories. It was possible to evidence that the professionals do not seem to have a satisfactory knowledge of patellar chondromalacia, since they presented superficial responses or that do not agree with the present literature. Conclusion: It is concluded that although post-rehabilitation is an area of important performance for the field of Physical Education, it still lacks the necessary knowledge for their performance, which may or may not aggravate the pathological condition of the client. From this perspective, future studies should be conducted to investigate Patellar Chondromyacia and other health / rehabilitation related injuries.
\end{abstract}

Keywords: Physical education professionals, Patellar hondromalacia, Post Rehabilitation.

1 Universidade Estadual de Montes Claros (UNIMONTES), Montes Claros-MG.

*E-mail: camilafernandaoliveira21@gmail.com

SUBMETIDO EM: 11/2019

ACEITO EM: 11/2019

PUBLICADO EM: $3 / 2020$

REAS/EJCH | Vol.12(5) | e2271 | DOI: https://doi.org/10.25248/reas.e2271.2020 


\section{RESUMEN}

Objetivo: Este estudio analizó las percepciones de los profesionales de Educación Física sobre la lesión de condromalacia rotuliana, así como la conducta clínica para el tratamiento de esta patología. Métodos: La investigación fue descriptiva y transversal, desarrollada por enfoque cualitativo. Doce profesionales que se graduaron del curso de Educación Física - Bachillerato, que trabajaron como instructor / personal en la ciudad de Montes Claros-MG, fueron entrevistados. La recolección de datos se realizó entre agosto y septiembre de 2019. El instrumento fue la entrevista individual con un guión semiestructurado, que se transcribió en su totalidad y, después de la transcripción y lectura de los informes, utilizando el método de categorización como recopilación de datos. Resultados: Las ideas de entrevista más relevantes fueron seleccionadas y agrupadas en cuatro categorías. Fue posible evidenciar que los profesionales no parecen tener un conocimiento satisfactorio de la condromalacia rotuliana, ya que presentaron respuestas superficiales o que no están de acuerdo con la literatura actual. Conclusión: Se concluye que aunque la posrehabilitación es un área de desempeño importante para el campo de la Educación Física, aún carece del conocimiento necesario para su desempeño, lo que puede agravar o no la condición patológica del cliente. Desde esta perspectiva, se deben realizar estudios futuros para investigar la condromiacia rotuliana y otras lesiones relacionadas con la salud / rehabilitación.

Palabras clave: Profesionales de la educación física, Condromalacia rotuliana, rehabilitación posterior.

\section{INTRODUÇÃO}

O ser humano necessita praticar exercício físico para favorecer uma boa qualidade de vida, pois quem se exercita regularmente tende a viver mais. Quando o corpo se torna inativo os músculos enfraquecem, o aumento da gordura corporal, entre outros e como consequência o corpo fica exposto a diversas doenças (NAHAS MV, 2013). Sendo assim, a Organização Mundial de Saúde (OMS) recomenda 20 minutos diários de atividade física leve ou moderada - totalizando cerca de 150 minutos por semana - para não ser considerado um indivíduo sedentário (MINISTÉRIO DA SAÚDE, 2017).

Segundo Ministério da Saúde (2017), no Brasil teve um aumento $7.3 \%$ na prática de atividades físicas de 2009 a 2016, essa crescente procura por ambientes que promovem a prática de exercício físico, seja por motivos de saúde, estética, melhoria da qualidade de vida, entre outros motivos, potencializam-se as possibilidades de atuação do profissional de educação física Bacharelado, em diferentes áreas de atuação como: exercícios físicos; desportos; musculação; recreação; reabilitação; dentre outras. Neste sentindo, ao atuar em qualquer cenário, este profissional deverá apresentar a competência técnica e humana para atender as necessidades e objetivos dos seus clientes (CONFEF, 2010).

Uma das áreas que vem crescendo bastante e com um público extremamente diversificado é a musculação ou treinamento resistido, são exercícios onde o movimento executado é o contrário da força exercida, gerando uma contração muscular (AZEVEDO RA, et al., 2012; WAGNER E, 2013) e, como qualquer exercício físico e até mesmo as atividades da vida diária que traz benefícios também pode causar danos à saúde, como as lesões musculoesqueléticas.

Se põe à prova se os profissionais estão preparados ou não, para a prescrição de exercícios individualizados e sistematizados, de acordo com a necessidade de cada cliente. Segundo Oliveira LC (2017), as pessoas podem ter a saúde e qualidade de vida preservada através da prática de exercícios físicos, e as que possuem algum tipo de lesões musculoesqueléticas, podem ser melhoradas, como a lesão Condromalácia Patelar que é uma lesão localizada na região anterior do joelho, sendo um desgaste na cartilagem da patela; o amolecimento da cartilagem sob a patela; na qual ocorre uma alteração angular ou rotacional da perna que desequilibra elementos do quadríceps e causa um desalinhamento patelar durante 0 movimento flexoextensao do joelho (SHERRY DD; PESSLER F, 2014).

Portanto, os estudos acima relatam a importância e o aumento na adesão na prática de exercício físico para garantir uma boa qualidade de vida. Sendo assim, determinados ambientes como, as academias de musculação exigem uma demanda de profissionais de educação física que sejam capacitados para auxiliar e prescrever exercícios físicos e, com conhecimentos sobre lesão para melhor atender os clientes/pacientes na 
prevenção ou na pós-reabilitação de lesões esportivas ou de atividades da vida diária. Esse estudo teve como objetivo analisar a percepções dos profissionais de educação física sobre a lesão Condromalácia Patelar, bem como a conduta clínica para o tratamento desta patologia.

\section{MÉTODOS}

A presente pesquisa pode ser classificada como descritiva e de corte transversal, e foi desenvolvida por meio da abordagem qualitativa. Participaram doze profissionais de educação física Bacharelado de ambos os sexos; das academias da região central da cidade de Montes Claros-MG. Para inclusão na pesquisa, os participantes deveriam ser formados em educação física Bacharelado; terem no mínimo 2 anos de atuação na área de personal trainer e/ou instrutor de musculação; possuir o documento do Conselho Regional de educação física - CREF; e assinarem o Termo de Consentimento Livre e Esclarecido.

A coleta dos dados foi realizada nos meses de agosto e setembro de 2019 e, buscando atingir o objetivo proposto, foi utilizada como instrumento de coleta de dados a entrevista individual com roteiro semiestruturado. Para o registro das entrevistas, foi utilizado um gravador a fim de gravar, para posterior análise, todas as informações fornecidas pelos participantes. Para a análise dos dados, foi utilizado o método da categorização, onde as categorias são empregadas para estabelecer classificações.

Por se tratar de uma pesquisa envolvendo seres humanos, o projeto foi submetido a um Conselho de Ética em Pesquisa da Universidade Estadual de Montes Claros que foi aprovado mediante Parecer Consubstanciado ํㅜ 3.331 .518 .

O estudo respeitou os princípios e as exigências formais contidas em normas que regulam as pesquisas envolvendo seres humanos. O aceite da participação se deu através da assinatura do Termo de Consentimento Livre e Esclarecido. Para manter o sigilo dos profissionais foram identificados pela inicial $(E)$ e o número de identificação.

\section{RESULTADOS E DISCUSSÕES}

Entre os 12 profissionais entrevistados de ambos os sexos, todos formados em educação física Bacharel com o tempo de formação entre 3 a 14 anos. O tempo de atuação como instrutor e/ou Personal Training variava de 3 anos e meio a 12 anos, e todos profissionais possuíam CREF. A maioria dos entrevistados não possuía especialização ou curso na área de lesões. As ideias consideradas mais relevantes, advindas da entrevista semiestruturada, foram selecionadas e agrupadas em quatro categorias.

\section{Conceito de Lesão Condromalácia Patelar}

Prentice WL e Voight ML (2003) expõem que a lesão Condromalácia Patelar consiste no amolecimento e deterioração da cartilagem articular na parte posterior da patela. Alguns fatores que podem desencadeá-la, como: o aumento do ângulo do quadríceps; inabilidade do músculo Vasto Medial Oblíquo; a postura do joelho (valgismo), o posicionamento do pé; traumas, entre outros; e há maior incidência em mulheres (STARKEY C e RYAN J, 2001; TAVARES GM, et al., 2011). Ao analisarmos as respostas de grande parte dos profissionals de educação física, parece que falta conhecimento mais profundo sobre esta lesão. Logo, verificamos algumas falas que mais se destacaram:

"É uma lesão no joelho que acaba sobrecarregando, acomete muito mulheres por causa geralmente do peso quadril, desvio de joelhos... joelho valgo". (E1)

"Pra mim, pelo que eu tenho conhecimento é uma lesão que é nas cartilagem, né? Que fica entre a patela e o fêmur e a tíbia. Então tem um desgaste nessa cartilagem, ai ocorrem as crepitações, tudo mais e dor." (E2).

Assim, percebemos que parece haver um conhecimento de forma superficial sobre o que é a lesão, faltam alguns pontos importantes, como questão anatômica, biomecânica e as suas características no que se diz a patologia. A lesão Condromalácia Patelar também conhecida como Dor Femoropatelar é uma patologia musculoesquelética, que acomete mais mulheres. Caracteriza-se por uma dor na região retropatelar; 
desalinhamento interno; amolecimento da cartilagem hialina; a biomecânica alterada como a diminuição flexoextensão do joelho; fraqueza nos músculos do quadril, principalmente o Vasto Medial Oblíquo (WILLY RW, et al., 2019). Já na fala abaixo o entrevistado parece nem saber o conceito desta patologia, apresentando até um pouco de desespero ao tentar responder.

\title{
"Desgaste da patela... (Silêncio) Mais? O meu pai do céu (Silêncio e risos)" (E3)
}

Ressaltamos então, como é complexo, no campo de atuação teórica-prática, o profissional, talvez, não apresentar conhecimento aprofundado sobre esta lesão, já que a saúde dos clientes está em jogo. Desta forma, Silva JM e Silva UGS (2012) entende que a lesão Condromalácia Patelar é uma pressão ou atrito entre o fêmur e a patela, que causa um desgaste na cartilagem. Neste sentido os profissionais, primeiramente, precisam ter entendimento sobre o que está lesão, para posteriormente, através de uma avaliação física minuciosa, direcionar o treinamento.

\section{As condutas realizadas pelo profissional de educação física na pós-reabilitação de um cliente/paciente com Condromalácia Patelar.}

O tratamento e as condutas devem ser conservadores, respeitando as limitações causadas por esta lesão, pois como não possui cura, é necessário que a pessoa esteja em constante fortalecimento/alongamento de algumas estruturas musculares específicas, para amenizar os desconfortos e dores causados. Realizando um trabalho de fortalecimento na musculatura do quadríceps e alinhar a patela (FERREIRA CLS, et al.,2008 ;MONNERAT E, et al., 2010).

A maioria dos entrevistados relatou que a primeira conduta realizada é a avaliação física para depois começar o processo de fortalecimento, pois permite conhecer melhor o cliente e o quadro/ grau da lesão. Alguns disseram fazer o teste de amplitude de movimento, para ter conhecimento do ângulo do exercício e o limiar da dor.

Segundo Batista LH et al. (2006), na avaliação física deve ser realizado o teste de amplitude de movimentos articular e a marcha. Permitindo assim delimitar a amplitude do movimento para melhor executar o exercício e a evolução do quadro. Abaixo veremos algumas falas com as condutas adotadas.

\begin{abstract}
"...faço avaliação, anamnese, pergunto como é que foi a fisioterapia, [...] e dependendo da resposta do tratamento eu praticamente começo do zero. Trabalho fortalecimento primeiro (com caneleira), fortalecimento específico do joelho, pra depois passar pra aparelho com cargas mais pesadas, [...] agachamento. Primeiro eu trabalho toda mecânica, dos movimentos, pra depois passar o exercício em si..." (E3).

"...Avaliação física, né? Primeiro de tudo, a gente monta programa, né? E a gente procura trabalhar sempre em cima da limitação que ele aluno tem [...] O tipo do protocolo ou tipos dos exercícios que a gente vai fazer a nível dos exercícios vai depender do grau de cada um, entendeu? [...] Ás vezes a pessoa chega com já tá sentindo uma crise, tá sentindo uma dor, aí a gente tem que ser um pouco mais conservador, né?..." (E4)
\end{abstract}

Os profissionais E3 e E4, não relatam sobre realizar o teste de amplitude; não especificam os músculos que precisam ser fortalecidos e alongados. Sendo essencial o fortalecimento do Vasto Medial Oblíquo e o alongamento de posteriores de coxas, como banda íleo-tibial e isquiotibiais para a melhora da patologia é necessário o equilíbrio entre os músculos agonistas e antagonistas da flexoextensão de joelho (SALDANHA DSA, et al., 2015; NASCIMENTO LR, et al., 2018). Já o E4 parece manter uma postura mais conservadora, mesmo não mencionando sobre os antecedentes de tratamento do seu cliente, de acordo com Muller TM (2006), antes de seguir na pós-reabilitação é importante os exames clínicos e físicos do individuo, pois é necessário conhecer o grau da lesão para prosseguir com a pós-reabilitação.

Já o profissional abaixo, relata que trabalha na prescrição de exercícios, ancorado pelas informações contidas no laudo do médico:

"...Com base no laudo médico encaminhado, passando ao processo de fortalecimento musculo-articular, tem como o condicionamento físico geral do indivíduo." (Ander). 
Porém, o Ander foi bem objetivo e não detalhou sobre as suas condutas, deixando transparecer, que possivelmente, não apresenta muito conhecimento sobre as condutas que devem ser realizadas na pósreabilitação, além do que vem recomendado no laudo do médico/fisioterápico. O fato de ter um encaminhamento médico, ou contato com o profissional da medicina e/ou fisioterapia, pode ser um fator decisivo para uma intervenção assertiva, por parte do profissional de educação física.

Estes diálogos sobre as condutas clínicas podem dar subsídios para uma intervenção pormenorizada e interessante. Entretanto, de acordo com o CONFEF Capítulo II (Do Campo e da Atividade Profissional) no Art. 9 - é função do Profissional de educação física prescrever exercícios na pós-reabilitação. Não cabe, portanto, a outro profissional, esta prescrição (que muitas vezes já vêm descritas em laudos médicos, por exemplo).

\section{Prescrição dos exercícios necessários para melhorar o quadro da lesão Condromalácia Patelar, na pós-reabilitação.}

Segundo Ferreira CLS et al. (2008) a prática da musculação pode trazer benefícios para melhora do quadro dessa patologia, pois irá reduzir o quadro de dor e progredir na amplitude do movimento. Mas de acordo com Roque $V$ et al. (2012), para prescrever os exercícios adequados é essencial ter um nível de conhecimento sobre a biomecânica da articulação femoropatelar. Alguns dos profissionais, possivelmente, não apresentam ter tanto conhecimento sobre os exercícios indicados para este tipo de tratamento. Como na fala do profissional E5 e E4 abaixo:

\section{" De início a cadeira extensora, não indicaria [...]."(E5)}

Esse aparelho é um dos indicados no tratamento, pois ativa o músculo Vasto Medial que precisa ser fortalecido nessa patologia, porém precisa ser realizado na angulação adequada. Segundo Lima CS e Pinto RS (2006) o ângulo que deve ser trabalhado é, exatamente, no final da extensão de joelho é de 0 a $30^{\circ}$. A realização da extensão do joelho também pode ser feita com caneleira que é mais eficaz, pois a resistência vai aumentando a cada ângulo final, sendo assim, ativando mais o Vasto Medial. Já Salvador BRC, et al. (2011) o fortalecimento na cadeira extensora pode ser realizado na angulação de 0 a $90^{\circ}$ ocorrendo variação a cada indivíduo.

"Não indicaria, éé cadeirinha na parede, agachamento com a bola nas costas, na parede, né? [...] Exercícios que tenha somente flexão éé extensão de pernas na cadeira extensora exercícios que envolva somente a extensão do joelho, [...]."(E4)

O E4 também parece ter pouco conhecimento sobre os exercícios para a Condromalácia Patelar, pois só foca em exercícios de extensão do joelho. Segundo Willy RM et al. (2019) os exercícios de extensão de joelho e agachamento, devem ser direcionados, pois ambos objetivam trabalhar a musculatura do joelho. Para Prentice WE e Voight ML (2003) diz que os exercícios de cadeia cinética fechada ajudam no fortalecimento do quadríceps e tendem amenizar as forças de reação da articulação femoropatelar. Desta forma são indicados, mas na angulação permitida como: Leg Press ( 0 a $\left.60^{\circ}\right)$, mini agachamento $\left(0\right.$ a $\left.40^{\circ}\right)$, subir degraus lateralmente utilizando um step de $20 \mathrm{~cm}$. O profissional abaixo relata que recebe laudos com algumas orientações sobre a prescrição dos exercícios de musculação:

"Quase $100 \%$ dos laudos que recebi direcionavam para o fortalecimento do vasto medial oblíquo. [...] creio eu o fortalecimento muscular do indivíduo seja o mais importante. Sem músculo trabalhar sozinho, a atuação sinergitas é o mais importante no meu ponto de vista. Exercícios Indicados: Cadeira Extensora e Cadeira adutora." (E6).

O entrevistado acima parece seguir apenas o que vem relatando no laudo médico. Sendo a assim, percebemos que pode haver uma insegurança de aplicar o seu conhecimento sobre a lesão, na hora de prescrever, e durante a entrevista se limita apenas em citar os exercícios adequados, que foram prescritos pelo médico. Desta forma, é importante ressaltar que o profissional habilitado para prescrever exercícios na pós-reabilitação de lesão, é apenas o Profissional de educação física. Os médicos devem apenas assinar um laudo indicando a patomecânica da lesão, e o profissional de educação física deve ter o conhecimento e discernimento para selecionar e prescrever os exercícios adequados a este tratamento (CONFEF 2010). 
Os respectivos relatos abaixo, os profissionais conseguem detalhar mais sobre os exercícios prescritos para a melhora do quadro da patologia. Sendo que os exercícios para melhora da lesão não devem ser aplicados somente Vasto Medial Oblíquo, mas, sendo indispensável o trabalho de fortalecimento e alongamento dos músculos agonistas e antagonistas da flexoextensão, para ter o equilíbrio articular femoropatelar (FERREIRA CLS, et al.,2008). Sendo assim, possivelmente, parecem ter mais estudos e/ou experiência em relação à prescrição para a lesão de Condromalácia Patelar, apesar de alguns erros de biomecânica que são explicitados:

"Então, eu sempre acreditei que exercícios com angulação mais curta [...] mais recente, a gente tem já indicativos que exercícios com amplitudes completa, mais assim, a gente utiliza exercícios com caneleira de flexão de quadril estendido, é, com rotação de pé para trabalhar vasto medial, fortalecimento de posterior, glúteo máximo, glúteo médio pra tá, porque geralmente o problema, do joelho eles não vão está exatamente no joelho, ele pode estar relacionado a outras lesões que a pessoa pode ter[...] Desequilíbrio muscular. (E7)

Neste direcionamento o profissional aparenta ter conhecimento quando se diz a respeito da angulação, mas se limita ao descrever os tipos de exercícios que podem ser trabalhados nessa lesão. Outro ponto a ser destacado também é a rotação externa dos pés, no movimento de flexão de quadril, que não influencia no trabalho do Vasto Medial (LIMA CS e PINTO RS, 2006). Apesar da Condromalácia Patelar não ter uma causa específica, pode sim estar relacionada a outro problema como: o joelho valgo, aumento do ângulo $Q$, patela lateral, músculos enfraquecidos, possibilitando a ocorrência de desequilíbrio osteomuscular (MONNERAT E, et al., 2010). Já o profissional abaixo relata que:

"[...] os exercícios de mobilidade de joelho, de articulação de joelho, de quadril, alongamentos de estruturas que estão encurtadas, eu acho que ia melhorar muito e fortalecimento das estruturas que precisam ser, vasto que sempre vão tá prejudicados [...] eu trabalharia agachamento sim, dentro da angulação permitida ela não sentir dor, [...] trabalharia sim cadeira extensora [...] eu não deixaria de trabalhar nenhum aparelho desde que ela conseguisse realizar, [...] igual mesmo a cadeira extensora ela isola o quadríceps de uma maneira que nenhum outro exercício consegue isolar[...] então eu posso colocar uma caneleira [...] propriocepção no jump com extensão de joelho, então não deixaria de trabalhar nenhum tipo de exercício de coxa, né, de quadríceps, de isquiotibiais, de posterior de coxa de maneira nenhuma, eu acho que eu trabalharia todos no limite da dor do paciente." (E8)

Já nessa fala acima é interessante que o profissional relata trabalhar com diversos exercícios como: o agachamento. Para Gusmão TMR et al. (2015) o agachamento além de trabalhar quadríceps e o glúteo máximo, permite a estabilidade de todas as articulações envolvidas durante a execução e sendo ainda, funcional, para as atividades de vida diária. Os exercícios devem ser prescritos de acordo com o quadro da lesão e com a melhora da funcionalidade de cada individuo permitindo a progressão nos exercícios. Os alongamentos são de suma importância, pois a falta de flexibilidade influencia diretamente na patologia. Logo, os músculos posteriores de coxa como banda íleo-tibial e isquiotibiais devem ser alongados e agregados no treinamento (ROQUE V, et al., 2012).

Alguns profissionais mencionaram que para a melhora do quadro é necessária a constância na realização de exercícios. Desta forma o cliente vai ter uma vida normal, sem limitações, até mesmo sem as dores e praticando esportes. Neste sentido, Fonchini D et al. (2010) vem comprovar, que para melhoria do quadro da lesão Condromalácia Patelar a continuidade na realização de exercícios é essencial.

\section{Existência de contato entre os integrantes da equipe multiprofissional para tratamento da lesão Condromalácia Patelar.}

O tratamento ou prevenção de lesões deve ser interdisciplinar ou multiprofissional, pois não deve focar apenas em uma área, e eliminando as demais que também atuam nesse processo, pois será ineficiente (QUEIROZ E e ARAÚJO TCCF, 2009). 
As atividades conjuntas beneficiam o cliente e visa uma relação entre trabalho e interação (PEDUZZI M, 2001). Apesar da importância do trabalho multiprofissional a maioria dos relatos dos entrevistados foi que parece não existir essa relação, denotando que alguns profissionais não conseguiam conversar entre si, mesmo que não invada a área do outro, como podemos ver na fala de Nadia:

"Era somente laudo, a gente não chegava entrar em contato com eles nã." (E1)

Percebe-se também na fala acima que os profissionais não se interagem, e não fazem esforços para que aconteça essa relação, afetando assim a realização do trabalho multiprofissional eficaz para o paciente/ cliente. Já o profissional E8, parece ter um contato com o fisioterapeuta/ médico, mas deixa transparecer que o trabalho de ambos não se interage permitindo assim o bem-estar do cliente/paciente.

A fala chama atenção uma vez que mesmo relata que o profissional "joga a bomba" para o outro, mas isso não é interessante, pois ele como um bom profissional deveria buscar ter esse diálogo, para conseguir realizar um trabalho de qualidade atendendo os objetivos do cliente. Os profissionais devem ser capazes de conjuntamente identificar as pontes positivos de ambos (CASANOVA IA, et al., 2015).

"Normalmente. Não só por Condromalácia [...], não, na verdade o que eu sinto é que eles (fisioterapeuta/ médico) meio que largam a bomba, vamos colocar assim $[\ldots] "$ (E8)

A entrevistada 9 narrou possuir um contato direto com outros profissionais, ocorrendo um trabalho em conjunto, interação profissional, permitindo troca de ideias. Percebe-se que esse trabalho multiprofissional traz muitos benefícios não apenas para o cliente, mas para os próprios profissionais envolvidos. $O$ trabalho e o aprendizado compartilhado por ambos consistem no crescimento de conhecimento (CASANOVA IA, et al., 2015).

"Direto, com o ortopedista e fisioterapeuta. Todas as recomendações né, opinião dele, a gente troca ideia como é que ele quer que a gente trabalha [...] então a gente trabalha em conjunto, é um trabalho multidisciplinar" (E9)

O trabalho multiprofissional deve ser realizado com a interação, trocando as informações do cliente. Mas sempre respeitando; e buscando o conhecimento (CASANOVA IA, et al., 2015). Portanto, percebemos a importância que o profissional precisa estar se atualizando, tendo constância em buscar o conhecimento, através de novos estudos científicos e cursos sobre a lesão, as suas condutas e os exercícios.

\section{CONSIDERAÇÕES FINAIS}

Esta pesquisa apresentou resultados importantes, pois mostrou que os profissionais parecem não ter tanto conhecimento sobre o que é a Condromalácia Patelar e a sua prescrição. Aparentam que também não há interação multiprofissional. Este trabalho nos chama atenção uma vez que a área de pós-reabilitação é uma área de suma importância para os Profissionais de educação física, pois exige conhecimento, responsabilidade e ética. Mostrando uma perspectiva positiva para a continuidade das pesquisas com 0 objetivo de investigar sobre uma percepção de Condromalácia Patelar e outras lesões em diversas situações de pesquisa científica relacionadas à saúde/reabilitação.

\section{REFERÊNCIAS}

1. AZEVEDO RA, et al. Exercício físico durante a gestação: uma prática saudável e necessária. Universitas: Ciências da Saúde, Brasília, 2011; 9(2): 53-70.

2. BATISTA LH, et al. Avaliação da amplitude articular do joelho: correlação entre as medidas realizadas com o goniômetro universal e no dinamômetro isocinético. Rev. bras. fisioter. [online], 2006; 10(2): 193-198.

3. CASANOVA IA, et al. Formação para trabalho em equipe na residência multiprofissional em saúde. ABCS Health Sci, 2015; 40(3): 229-233.

4. CONFEF. 2010. Estatuto do Conselho Federal de educação física. Disponível em: https://www.confef.org.br/confef/conteudo/471. Acesso em: 01 abr. 2019. 
5. FERREIRA CLS, et al. Efeitos dos exercícios de musculação para o fortalecimento da musculatura da coxa em portadora de condromalácia patelar. Coleção pesquisa em educação física, Rio de Janeiro, 2008; 7(3): $223-228$.

6. FOSCHINI D, et al. Treatment of obese adolescents: the influence of periodization models and ACE genotype. Obesity, São Paulo, 2010; 18(4): 766-772.

7. GIL AC. Métodos e técnicas de pesquisa social. 6ed. São Paulo: Atlas, 2008; 220p.

8. GUSMÃO TMR, et al. Desempenho funcional do exercício de agachamento. Caderno de Graduação - Ciências Biológicas e da Saúde, 2015; 2(3): 45-56.

9. LIMA CS, PINTO RS. Cinesiologia e Musculação. Porto Alegre: Artmed, 2006; 188p.

10. MINAYO MCS. O desafio do conhecimento: Pesquisa qualitativa em saúde. 11. Ed. São Paulo, SP: HUCITEC, 2008.

11. MINISTÉRIO DA SAÚDE. 2017. Atividade física. Disponível em: http://portalms.saude.gov.br/component/content/article/781atividades-fisicas/40390-atividade-fisica. Acesso em: 01 abr. 2019.

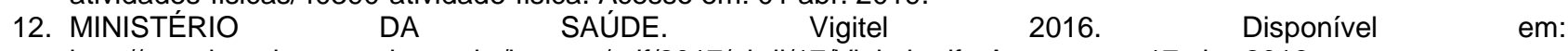
http://portalarquivos.saude.gov.br/images/pdf/2017/abril/17/Vigitel.pdf. Acesso em: 17 abr. 2019.

13. MONNERAT E, et al. Abordagem fisioterapêutica em pacientes com condromalácia patelar. Fisioterapia. Ser.Grajaú, 2010; 5(1): 57-60.

14. MULLER TM. Estudo da eficácia da hidrocinesioterapia no tratamento da artrose de joelho. (Monografia) Graduação em Fisioterapia Bacharel. Faculdade Assis Gurgacz, Cascavel, 2006; 100p.

15. NAHAS MV. Atividade Física, Saúde e Qualidade de Vida: Conceitos e sugestões para um estilo de vida ativo. 6 a . Londrina: Midiograf, 2013.

16. NASCIMENTO LR, et al. Hip and Knee Strengthening Is More Effective Than Knee Strengthening Alone for Reducing Pain and Improving Activity in Individuals with Patellofemoral Pain: A Systematic Review with Meta-analysis. Journal of Orthopaedic \& Sports Physical Therapy, 2018; 48(1): 19-31.

17. OLIVEIRA LC. Avaliação, proposta de tratamento e intervenção fisioterapêutica em um paciente com aderência cicatricial no joelho. Fisioterapia Brasil, São José do Rio Preto, 2017; 12(2): 121-126.

18. PEDUZZI M. Equipe multiprofissional de saúde: conceito e tipologia. Rev Saúde Pública. 2001; 35(1): $103-109$.

19. PRENTICE WE, VOIGHT ML. Técnicas em reabilitação musculoesquelética. Trad. Terezinha Oppidi e Maria Alice Quartim Barbosa de Araújo. Porto Alegre: Artmed, 2003; 727p.

20. QUEIROZ E, ARAÚJO TCCF. Trabalho em equipe em reabilitação: um estudo sobre a percepção individual e grupal dos profissionais de saúde. Paideia, 2009; 19(43): 177-187.

21. ROQUE V, et al. Síndrome Femoro-Patelar. Revista da Sociedade Portuguesa de Medicina Física e de Reabilitação, 2012; 22(2): 53-61.

22. SALDANHA DSA, et al. Hidrocinesioterapia na Condromalácia Patelar: Estudo de caso. Revista FAIPE, Cuiabá 2015; 5(1): 49-67.

23. SALVADOR BRC, et al. Comparação da eficácia de exercício de cadeia cinética aberta e fechada na síndrome dor patelo femural. Fisioterapia em Movimento, Curitiba, 2011; 24(1): 167-172.

24. SHERRY DD e PESSLER F. 2017. Condromalacia patelar. Disponível em: https://www.msdmanuals.com/pt$\mathrm{br} /$ profissional/pediatria/dist\%C3\%BArbios-do-tecido-conjuntivo-em-crian\%C3\%A7as/condromalacia-patelar. Acesso em: 20 mar. 2019.

25. SILVA JM, SILVA UGS. Benefícios de um programa de fortalecimento de quadríceps em pacientes acometidos por osteoartrite: uma revisão de literatura. (Monografia) - Graduação em Fisioterapia Bacharel. Faculdade Nobre de Feira de Santana, Bahia, 2012; 50p.

26. STARKEY C, RYAN J. Avaliação de Lesões Ortopédicas e Esportivas. 1ed. Manole, 2001; 582p.

27. TAVARES GM, et al. Condromalácia Patelar: análise de quatros testes clínicos. ConScientiae Saúde, 2011; 10(1): 77-82.

28. TEIXEIRA LS, et al. Corrente Excitomotora e Força Muscular em Reabilitação: Revisão de Literatura. EFDEPOTES.COM. Revista Digital, Buenos Aires, 2008; 121.

29. TURATO ER, et al. Amostragem por saturação em pesquisas qualitativas em saúde: contribuições teóricas. Cad. Saúde Pública, Rio de Janeiro, 2008; 24(1): 17-27.

30. WAGNER E. Estudo de lesões musculares e articulares em praticantes de musculação de uma academia do município de Florianópolis-SC. (Monografia). Federal de Santa Catarina, Florianópolis-SC, 2013; 52p.

31. WILLY RW, et al. Patellofemoral Pain: Clinical Practice Guidelines Linked to the International Classification of Functioning, Disability and Health From the Academy of Orthopaedic Physical Therapy of the American Physical Therapy Association. J Orthop Sports Phys Ther, 2019; 49(9): 1-95. 